\title{
Threshold Models for Time Series of Counts: MRSA as A Case Study
}

\author{
Dr. Noelle Samia \\ Northwestern University, USA \\ n-samia@northwestern.edu
}

A threshold time-series model is a class of nonlinear time-series models, commonly used in areas such as economics. The usefulness of these advanced nonlinear techniques has not yet been exploited and used in the medical fields. In our paper, we demonstrate the importance and the information that can be elucidated from complex (nonlinear) datasets, using the threshold models for time series of count data, and we illustrate their usefulness in the area of infectious diseases and the biomedical field in general. Using a unique dataset of more than 1 million patients in acute care facilities across the USA, we provide statistical quantitative evidence on the role of healthcare-associated methicillin-resistant Staphylococcus aureus (MRSA) infections among other healthcare-associated infections (HAI). Our results are achieved by bridging the gap between medical/clinical physicians, theoretical epidemiologists, and statisticians. 